\title{
EFFECT OF PHASE OF HARMONICS UPON ACOUSTIC QUALITY.
}

\author{
By M. G. Lloyd and P. G. Agnew.
}

\section{INTRODUCTION.}

Ever since the celebrated controversy between Helmholtz and König concerning the influence of the phase of harmonics, this question has remained a point of dispute between the supporters of these two great experimenters. Each of them had conducted experiments which apparently supported his case, König finding that the quality was affected by phase displacement of a harmonic, while Helmholtz found no effect.

The method used by Helmholtz ${ }^{1}$ to produce a change in phase was elegant, but open to objection. He shaded the aperture of the resonator used to reenforce the sound. This changes the amplitude as well as the phase of the vibration, and necessitated a compensating correction by altering the distance between fork and resonator. It is thus evident that the change from one condition to another could not be made with much rapidity. König's ${ }^{2}$ experiments were made with the wave siren, which has since been shown to give small quantities of extraneous harmonics. ${ }^{3}$ His results are therefore of doubtful significance.

The experiments to be described made use of electrical currents whose wave form could be controlled, a telephone receiver being used to transform these into sound waves.

\section{APPARATUS.}

Definite composition of the electrical wave was made possible by the use of a special set of generators at the Bureau of Standards,

${ }^{1}$ H. L. F. Helmholtz, Sensations of Tone, 3d ed., p. I24.

${ }^{2}$ R. König, Quelques Experiences d'acoustique, p. 222.

${ }^{3}$ R. König, Wied. Ann. 57, p. 339; г896. C. Stumpf, Wied. Ann. 57, p. 677 ; 1896. 
known as the Harmonic Set. This set consists of two driving motors and eight three-phase generators, which at normal speed give frequencies of $60,180,300,420,540,660,780$, and 900 cycles per second. They are assembled in two sections, the generators with the three lower frequencies being mounted on the shaft of one motor, while the others are mounted with the second motor. The two sections can be coupled rigidly together when desired. Each generator has rotating poles and stationary unslotted armature, and the position of the armature can be shifted by means of a worm gearing and handwheel. By connecting the generators in series, and properly exciting the fields and setting the armatures, any odd harmonic up to the fifteenth can be superposed upon the fundamental frequency in any amplitude and phase relation, thus permitting the synthesis of an endless variety of wave forms. The individual generators give waves which are approximately sinusoidal, although containing a few per cent of harmonics.

Another generating set which has been used consists of two two-phase generators of 60 and r 80 cycles, respectively, connected to a driving motor. These machines give nearly pure sine waves. A third generator set furnishing three-phase and 60 cycles was also used.

Several telephone receivers were used, but most of the experiments were carried out with a Siemens \& Halske instrument of ro.5 ohms resistance. The telephone was sometimes used with a high resistance in series; at other times it was placed in parallel with an adjustable carbon resistance of low value, and the whole put in series with an incandescent lamp. Variation of the carbon resistance altered the current through the telephone. In a few of the experiments separate telephones were connected to the generators, and the sound waves combined.

\section{EXPERIMENTS.}

At first the simple method of armature shift was used. Several combinations gave changes in the note sounded. But the method was neither convenient nor very sensitive, several observers and precautions to avoid suggestion being deemed necessary to firmly establish the change as taking place. The changes could be detected only with particular percentages of harmonic. 
To shift the phase more quickly, a phase-shifting transformer was tried, but was found unsuitable, as slight changes in voltage and wave form accompanied the rotation of the secondary.

Finally, use was made of a second motor-generator set. By taking the fundamental from one and the harmonics from the other, and then running the machines just out of synchronism, we have one wave train slowly drifting relatively to the other, thus producing a slowly and periodically recurring change of phase.

The effect is much the same as in the arrangement used by Lord Kelvin, ${ }^{4}$ who took two tuning forks, one of which was an exact harmonic of the other, and changed its period very slightly by adding a small weight to it, thus giving a continuous cyclic change of phase.

TABLE I.

\begin{tabular}{|c|c|}
\hline Cycles & Changes per lamp cycle \\
\hline 60 & $\left\{\begin{array}{r}3 \\
15\end{array}\right.$ \\
\hline 300 & 15 \\
\hline 420 & 21 \\
\hline 540 & 9 \\
\hline 660 & 33 \\
\hline 780 & 39 \\
\hline 900 & 15 \\
\hline
\end{tabular}

This method was found to be very much more sensitive than either of the others. In fact, with the armatures connected single phase, every combination of harmonics tried showed distinct cyclic changes in the note sounded. By controlling the speed of one of the generators the change in phase may be made rapid or slow, the rate being observed by means of a synchronizing lamp connected in series with the 6o-cycle generator of each set. Relative intensities of the electromotive forces of the generators connected to the telephone could be adjusted by resistance in the field circuits. This method proved very convenient, the control being easy and flexible.

${ }^{4}$ W. Thomson, Proc. Roy. Soc. Edinburgh, 9, p. 602; 1878. 5249-No. 2-09-8 
Table I shows the results of using each frequency of the harmonic set with the I8o-cycle two-phase generator. Changes in quality were evident in every case, and the number of cyclic changes occurring during a complete cycle of the synchronizing lamp were counted. This is the number of changes taking place while one 6o-cycle generator gains a cycle upon the other. The significance of these numbers is seen when we consider the impurities present in the waves from the two generators. Thus, with 300 cycles, the possible overtones have frequencies of 900 , I 500 , 2100 , etc. The frequency of 900 cycles is common to both, and with shifting phase these components will at times oppose, at other times reenforce, each other, thus changing the amplitude of this component of the combination. As the wave of this frequency shifts in phase by a complete cycle for one-fifteenth of a cycle of the 6o-cycle generator, a shift of an entire cycle of the latter corresponds to a shift of fifteen entire cycles of the higher frequency, and consequently fifteen cycles of amplitude change. In each of the other cases it will be found similarly that the number of changes counted corresponds to some common harmonic of the two frequencies used. In one case (6o cycles) two distinct and recurring changes could be distinguished, each one depending upon a separate frequency, common to the two generators, namely, I 80 and 900 cycles. No doubt other frequencies are common to the two generators in every case, but are not prominent enough to be heard.

It is evident that the changes in quality observed are simply beats due to the interference of frequencies common to the two sources. For an experiment to be decisive it is necessary that the two sources should have no harmonic common to them. Lindig, ${ }^{5}$ in 1902 , found similarly that changes in quality always resulted when the two sources furnished the same harmonic. He used a telephone in conjunction with a telephone siren, and found no change in quality except when this condition was fulfilled.

Alternators giving rigorously sinusoidal waves are not obtainable. Yet it is well known that in three-phase generators the $3^{\text {rd, }}$ 9th, $15^{\text {th }}$, 2 Ist, etc., harmonics are eliminated automatically. It is to be noticed that with two machines so connected, if 
only frequencies having ratios of 3 to $\mathrm{I}$ and 9 to $\mathrm{I}$ are used, then there will be no common impurity, even though they are not necessarily sinusoidal. Again, in most generators the evennumbered harmonics are entirely lacking, so that if generators with frequencies in the ratio of 2 to I can be used there will again be no common impurity. The first condition was attained by using the two sections of the harmonic set separately, or by using one of them with a second 3-phase generator. The second arrangement was carried out by running one generator at half speed, which also allowed other combinations to be used, which should theoretically give no common harmonics.

It will be seen that we were thus enabled to test under the three distinct conditions, in which the phase of an overtone was constantly changing in respect to the fundamental, the overtone and the fundamental containing no common impurity, viz, an odd harmonic, an even harmonic, and an overtone which was not harmonic-that is, an overtone having a pitch ratio of $3 / 2,4 / 3$, or the like.

When the harmonic set was run in separate parts, an oscillograph was used as a synchronizing apparatus.

It should be said that a direct synthesis of sound waves by this method does not seem to be possible without introducing distortion.

When a separate telephone was excited from each source and the two placed inside the body of a mandolin to act as a sounding board and combine the sounds, very distinct changes were observed, often of more than one period. They could not be made to disappear by any arrangement of intensities, nor even to materially decrease. Evidently these changes of tone were chiefly due to the harmonics introduced by the sounding board, just as we should expect.

Accordingly, in the final experiments the emf. waves were directly combined and applied to the telephone. This method assumes that the diaphragm gives out a sound wave having the same form as the electrical wave impressed upon the telephone. To test this assumption, the telephone was excited from a starconnected generator, and the sound analyzed by a set of Helmholtz resonators ${ }^{6}$ in order to determine whether the sound wave

${ }^{6}$ These were very kindly loaned to us from the University of Pennsylvania by Prof. A. W. Goodspeed, to whom we are greatly indebted. 
given out by the telephone contained harmonics not present in the electrical wave.

The generator was run at such a speed that one of the resonators reenforced the fundamental, and the sound was then examined by means of resonators whose natural frequencies were two, three, four, and five times as great. Four different telephones were thus examined, the results being shown in Table II. The fifth harmonic was no doubt present in the electrical wave, whereas the second, third, and fourth may reasonably be assumed to be absent. It is to be noticed that the third was always present in the sound wave, whereas the second and fourth were sometimes absent or too weak to be heard. They are less prominent in the shunt than in the series connection, and are never strong in the Siemens \& Halske and Bell receivers.

\section{TABLE II.}

\section{Experiments with Resonators.}

Harmonic set, star-connected, and run slow, so that the 30o-cycle generator gave about 256 cycles. With small current in the telephone, such as to require pressing against ear, no harmonics discernible.

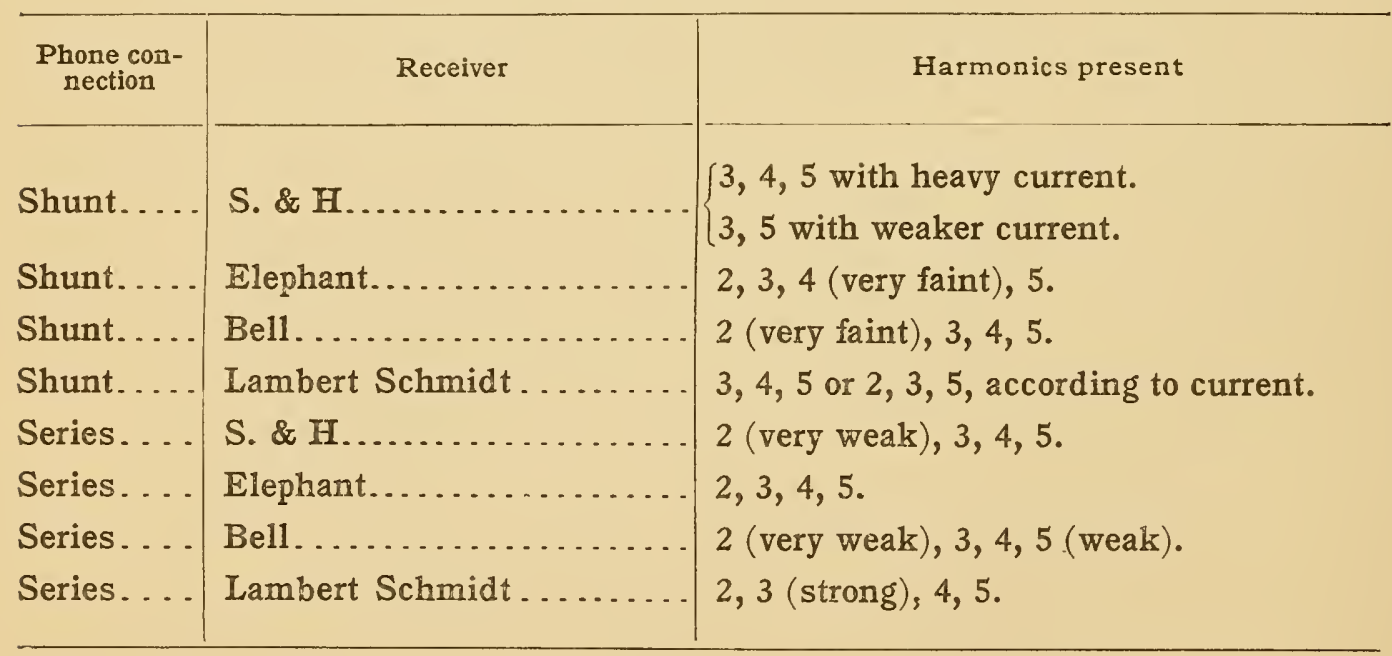

Summary: $3 \mathrm{~d}$ and 5 th always noticeable, and $3 \mathrm{~d}$ as strong as 5 th; $2 \mathrm{~d}$ and 4 th sometimes absent, or too weak to be heard, and less prominent in shunt than in series connection. The $2 \mathrm{~d}$ is never strong in the Siemens \& Halske nor in the Bell. 
It might seem that the distortion found by the resonators was introduced, not by the diaphragm, but by the armature reaction in passing a current from one machine through the armature of a second; but only a very small current was used, and the harmonic set is rated at 34.5 amperes. Moreover, if it were due to armature reaction, the distortion should be greater for the shunt connection, where a few tenths of an ampere were drawn, than in the series connection, where only micro-amperes were drawn; but the reverse was found.

It is thus evident that the receiver may introduce harmonics not present in the electrical wave, and thus lead to false inferences in regard to the question being studied. The best conditions are evidently obtained by using a sensitive receiver in shunt connection, excited as slightly as possible, and star-connected generators whose ratio of frequencies is an even integer. The frequencies should, moreover, be as high as possible, since the ear is more sensitive at higher frequencies and less current is necessary.

TABLE III.

\begin{tabular}{c|l}
\hline Pitches of components & Intensity of sound possible without beats \\
\hline $60-180$ & Less than normal. \\
$60-540$ & Less than normal. \\
$60-900$ & Less than normal. \\
$180-270$ & Normal. \\
$180-390$ & Norma1. \\
$180-450$ & Normal. \\
$180-540$ & Normal. \\
$300-450$ & Normal. \\
$300-900$ & Above normal. \\
\hline
\end{tabular}

In the final experiments at least one of the machines used was star connected, three phase. Even under the most favorable conditions of connection every combination tried could, by using sufficient current in the telephone, be made to show a periodic change of quality which gave the same sensation to the ear as occurred in the case of the single-phase connection described above. This could be done with the telephone either in series or in shunt, and with five different types of telephone. Lindig does not mention 
this effect with strong tones. Perhaps the greater sensitiveness of the continuous slip-phase method accounts for it being noticed in our experiments and not in his.

Yet in nine different cases it was found that with less current in the telephone no change could be detected by either observer. These are shown in Table III.

By "normal" is here meant an intensity that would allow spoken words to be easily and distinctly understood, but not great enough to make the sound unpleasant-that is, about the intensity of ordinary conversation over the telephone.

In these cases it is probable that a change in quality as great as would be caused by the interference of harmonics common to the two sources, when present to the amount of even less than I per cent, could have been easily detected.

But in several cases beats were obtained which could not be made to disappear by decreasing the intensity of the sound, and this when, theoretically, there should have been no common harmonics present, and as far as could be told the machines were running smoothly. For example, using the two-phase machine with the harmonic set, beats were obtained with the following combinations:

$$
\begin{array}{r}
90-180 \\
\text { I } 80-270 \\
\text { I } 80-45^{\circ}
\end{array}
$$

Yet it will be noticed that, as indicated in the previous table, the last two combinations gave no beats when the two parts of the harmonic set were used as source. A little light may be thrown upon the discrepancy by a consideration of the second case. The r 8o-cycle emf. from the two-phase machine is known to contain 4 per cent of its third harmonic, corresponding to a pitch of 540 . Now, if the telephone diaphragm introduced this tone independently its amplitude would be likely to be altered by the addition of this harmonic to the electrical wave or by a change in its phase relation to the fundamental. In other words, the distortion of the wave during its conversion from the electrical to the mechanical form would naturally vary with the form of the electrical wave, and particularly with any alteration in those components which are independently introduced during the conversion. Evidently these results should be discarded in favor of those obtained 
under the more favorable conditions of taking both the components from star-connected machines. When so connected, and when both machines were running smoothly under the best conditions, no case of a change in quality was found that could not be traced to extraneous causes.

Very great care had to be observed to exclude such extraneous interference, and in many cases it was found to be impossible to do so. Several times during the course of the work results were obtained that seemed to indicate a real dependence of quality upon phase, but in each case investigation showed that the results were vitiated by bad conditions. For example, the throbs of the engine supplying power to the dynamo, which in turn supplied the direct current to the driving motor, could occasionally be heard in the telephone, and for this reason nearly all the observations were made while the energy was being taken from a storage battery. Noises in the dynamo room were sometimes transmitted through the telephone, and slip rings and commutators had to be in perfect condition or the sound became unsteady. Mechanical beats were sometimes transmitted to the telephone, as, for example, when it was attempted to obtain the combination $300-600$ by running the halves of the harmonic set at slightly different speeds, the note given out by the telephone when connected to the $300-$ cycle machine alone contained beats which ceased when the machine of higher frequency was stopped. Unfortunately this condition prevented us from getting results with nearly all of the combinations involving even harmonics.

Thus, while the method is very sensitive, positive results should be accepted only after care has been taken to insure that no disturbing factors are present. The ideal condition for its use would be to have the machines in separate buildings and the observer in a third.

\section{SUMMARY.}

The experiments clearly support Helmholtz's view that quality is independent of phase relations, as was found by Lord Kelvin with his analogous mechanical drifting phase method, and also show that the telephone introduces both odd and even harmonics which are not present either in the emf. wave at its terminals, or in the current wave through it.

WASHINGTON, June 30, I909. 